\section{MRSA sensitivity training}

\section{By Tim Fulmer, Senior Writer}

Researchers at Merck \& Co. Inc. have identified targets in methicillinresistant Staphylococcus aureus that synergize with $\beta$-lactam antibiotic targets to restore antibiotic sensitivity. ${ }^{1}$ The findings suggest it may now be possible to use combinations of antibiotics to treat infections previously considered intractable.

Methicillin-resistant $S$. aureus (MRSA) infections are the most frequent cause of skin and soft tissue infections seen in the emergency room in the U.S. In addition, MRSA accounts for about $20 \%$ of bloodstream infections in hospital settings. ${ }^{2}$

MRSA is unresponsive to common $\beta$-lactam antibiotics, including penicillin and imipenem, and can develop resistance to antibiotics of last resort such as vancomycin.

The basic mechanism of MRSA resistance to $\beta$-lactam antibiotics has been known for more than 20 years. S. aureus synthesizes a new bacterial wall by means of several enzymes, including transpeptidases in the penicillin binding protein (PBP) family. Over time, some strains have evolved PBPs such as penicillin binding protein 2A (PBP2A) that have a very low affinity for standard $\beta$-lactam antibiotics, rendering the bacteria resistant to these drugs. ${ }^{3,4}$ Efforts to target that mechanism have been stymied by the difficulty of designing compounds that bind PBP2A and are not degraded by other bacterial pathways. ${ }^{5}$

The Merck researchers took a different tack. Rather than target the resistance mechanism directly, they hypothesized that it might be possible to resensitize MRSA to $\beta$-lactam antibiotics by combining them with a second agent targeting a protein that is essential to overall bacterial function. By blocking a key cellular process such as cell division or cell wall synthesis, the second agent would compromise the overall viability of MRSA and potentially increase its susceptibility to the $\beta$-lactam antibiotic.

The Merck group had previously reported the design of an in vitro screen to identify MRSA targets that could be inhibited in combination with $\beta$-lactam antibiotics. ${ }^{6}$ Now, the group applied the screen to a range of $\beta$-lactam antibiotics.

The screen consisted of a panel of 43 MRSA strains, each of which differed from wild-type MRSA by having a single gene knocked down. The strains were then treated with 12 different $\beta$-lactam antibiotics to see if any combination of drug plus gene knockdown led to increased MRSA death.
The screen identified 13 knockdown strains that had greater rates of MRSA death following antibiotic treatment than the other 30 . The 13 included strains lacking genes encoding proteins involved in peptidoglycan biosynthesis, cell wall biosynthesis, signal transduction pathways and cell division.

Among those, three strains deficient in the cell division proteins FtsZ, FtsA and FtsW showed some of the highest MRSA death rates following treatment with generic $\beta$-lactam antibiotics such as imipenem, ertapenem and tazobactam.

Those results suggested that pairing a $\beta$-lactam antibiotic with an inhibitor of one of the three cell division proteins could be a way to treat MRSA.

To test that hypothesis, the researchers combined imipenem with the small molecule FtsZ inhibitor PC190723 and treated a mouse model of MRSA infection of the thigh.

The combination significantly decreased MRSA infection compared with vehicle $(p<0.05)$, whereas either agent alone failed to show a significant effect.

"Collectively, these data support a target-based approach to rationally develop synergistic combination agents that mitigate drug resistance and effectively treat MRSA infections," the authors wrote in their Science Translational Medicine paper describing the results.

"It is the synergy between the two compounds that is surprising and might, for example, lead to potentially resurrecting penicillin compounds for MRSA," said Simon Tucker, VP of research at Biota Holdings Ltd.

\section{Next steps}

Team leader Terry Roemer, biology lead of antibacterials at Merck Research Laboratories, told SciBX his group will continue to use the screening strategy to identify other MRSA targets. "The goal is developing new combination agents that display favorable characteristics of synergy and suppress drug resistance to treat MRSA and MRSE [methicillin-resistant S. epidermis]," he said.

Roemer also said he plans to develop "new antibiotic leads" against FtsZ.

The FtsZ inhibitor used in the paperPC190723-was synthesized in 2008 by Prolysis Ltd. That year, the company published in Science that PC190723 had in vitro activity against a variety of Staphylococcus strains. ${ }^{7}$ In 2009, Biota acquired Prolysis for \$10 million in stock.

Since that time, Merck researchers and others have synthesized PC190723 based on information in the public literature and have used the compound as a research tool in a variety of studies. ${ }^{8}$

Biota is optimizing next-generation FtsZ inhibitors to treat Staphylococcus infections, said Tucker. Those new inhibitors are of a different chemical class than PC190723, although Tucker did not provide further details.

Richard Scott, VP of research at PolyMedix Inc., said it will be important to identify other bacterial targets that synergize with $\beta$-lactam 


\section{ANALYSIS}

antibiotics "because not all MRSA strains show synergy between PC190723 and imipenem, and there is a relatively high frequency of resistance with FtsZ."

In the Science Translational Medicine paper, the Merck researchers did find a number of S. aureus strains that were resistant to PC190723. As expected, genetic analysis of those strains indicated the resistance resulted from spontaneous mutations in the FtsZ protein.

PolyMedix's lead compound is PMX-30063, a synthetic defensin mimetic antibiotic that is in Phase II trials to treat acute bacterial skin and skin structure infections caused by $S$. aureus. First data are expected in $1 \mathrm{H} 12$.

Merck's findings are covered by a patent.

Fulmer, T. SciBX 5(14); doi:10.1038/scibx.2012.351

Published online April 5, 2012

\section{REFERENCES}

1. Tan, C.M. et al. Sci. Transl. Med.; published online March 21, 2012; doi:10.1126/scitranslmed.3003592

Contact: Terry Roemer, Merck Research Laboratories, Kenilworth, N.J.

e-mail: terry_roemer@merck.com

2. Klevens, R.M. et al. JAMA 298, 1763-1771 (2007)

3. Hartman, B.J. \& Tomasz, A. J. Bacteriol. 158, 513-516 (1984)

4. de Lencastre, H. et al. Curr. Opin. Microbiol. 10, 428-435 (2007)

5. Moreillon, P. Clin. Microbiol. Infect. 14, 32-41 (2008)

6. Lee, S.H. et al. Chem. Biol. 18, 1379-1389 (2011)

7. Haydon, D.J. et al. Science 321, 1673-1675 (2008)

8. Andreu, J.M. et al. J. Biol. Chem. 285, 14239-14246 (2010)

COMPANIES AND INSTITUTIONS MENTIONED

Biota Holdings Ltd. (ASX:BTA), Notting Hill, Victoria, Australia Merck \& Co. Inc. (NYSE:MRK), Whitehouse Station, N.J. PolyMedix Inc. (OTCBB:PYMX), Radnor, Pa. 